\title{
QTL identification, fine mapping, and marker development for breeding peanut (Arachis hypogaea L.) resistant to bacterial wilt
}

\author{
Feiyan Qi ${ }^{1}$ Ziqi Sun ${ }^{1} \cdot$ Hua Liu ${ }^{1} \cdot$ Zheng Zheng $^{1} \cdot$ Li Qin $^{1} \cdot$ Lei Shi ${ }^{1}$ - Qingzheng Chen ${ }^{2} \cdot$ Haidong Liu $^{2} \cdot$ Xiufang Lin $^{2}$. \\ Lijuan Miao ${ }^{1} \cdot$ Mengdi Tian ${ }^{1} \cdot$ Xiao Wang $^{1} \cdot$ Bingyan Huang ${ }^{1} \cdot$ Wenzhao Dong $^{1} \cdot$ Xinyou Zhang $^{1}{ }^{\circledR}$
}

Received: 31 July 2021 / Accepted: 31 December 2021 / Published online: 20 January 2022

(c) The Author(s) 2022

\begin{abstract}
Key message A major QTL, qBWA12, was fine mapped to a $216.68 \mathrm{~kb}$ physical region, and A12.4097252 was identified as a useful KASP marker for breeding peanut varieties resistant to bacterial wilt.

Abstract Bacterial wilt, caused by Ralstonia solanacearum, is a major disease detrimental to peanut production in China. Breeding disease-resistant peanut varieties is the most economical and effective way to prevent the disease and yield loss. Fine mapping the QTLs for bacterial wilt resistance is critical for the marker-assisted breeding of disease-resistant varieties. A recombinant inbred population comprising 521 lines was used to construct a high-density genetic linkage map and to identify QTLs for bacterial wilt resistance following restriction-site-associated DNA sequencing. The genetic map, which included 5120 SNP markers, covered a length of $3179 \mathrm{cM}$ with an average marker distance of $0.6 \mathrm{cM}$. Four QTLs for bacterial wilt resistance were mapped on four chromosomes. One major QTL, $q B W A 12$, with LOD score of 32.8-66.0 and PVE of $31.2-44.8 \%$, was stably detected in all four development stages investigated over the 3 trial years. Additionally, $q B W A 12$ spanned a $2.7 \mathrm{cM}$ region, corresponding to approximately $0.4 \mathrm{Mb}$ and was fine mapped to a $216.7 \mathrm{~kb}$ region by applying KASP markers that were polymorphic between the two parents based on whole-genome resequencing data. In a large collection of breeding and germplasm lines, it was proved that KASP marker A12.4097252 can be applied for the marker-assisted breeding to develop peanut varieties resistant to bacterial wilt. Of the 19 candidate genes in the region covered by $q$ BWA12, nine NBS-LRR genes should be further investigated regarding their potential contribution to the resistance of peanut against bacterial wilt.
\end{abstract}

\section{Introduction}

Cultivated peanut (Arachis hypogaea L.) is one of the most important oil crops; its worldwide yield (up to 47 million tons) ranked 4th after soybean, canola, and sunflower. The top 10 countries produced $82.5 \%$ of the global peanut yield,

Communicated by Henry T. Nguyen.

Feiyan Qi and Ziqi Sun have contributed equally to this study.

Xinyou Zhang

haasz@126.com

1 Henan Academy of Crop Molecular Breeding, Henan Academy of Agricultural Science/Key Laboratory of Oil Crops in Huang-Huai-Hai Plains, Ministry of Agriculture/ Henan Provincial Key Laboratory for Oil Crop Improvement, Zhengzhou 450002, Henan, China

2 Hezhou Academy of Agricultural Science, Hezhou 542899 , Guangxi, China while most of these countries are in tropical and subtropical areas, where bacterial wilt is a major peanut disease (Xu et al. 2009; Norman et al. 2009). Peanut bacterial wilt is caused by Ralstonia solanacearum, which also causes potato brown rot, bacterial wilt in tomato, tobacco, eggplant, and some ornamental plants, and Moko disease in banana (Paudel et al. 2020). Ralstonia solanacearum ranks second among the top 10 pathogens in plant pathology because it is widely distributed and is highly pathogenic (Mansfield et al. 2012). Peanut bacterial wilt had been found in most areas of the 13 major peanut-producing provinces in China, and the disease was estimated to affect 800,000 hectares of agricultural land, accounting for nearly $16 \%$ of the total agricultural land in the country (Jiang et al. 2017). This disease can lead to $10-20 \%$ peanut yield loss, but in severe cases, yield loss may be as high as $100 \%$. In the last decade, bacterial wilt began to spread from Southern China to the cool uplands at higher latitudes in Northern China. Changes in farming systems and soil treatments resulted in minimal 
improvements in controlling bacterial wilt (Yu 2011; Jiang et al. 2017). Thus, cultivating disease-resistant peanut varieties is the most economical and effective way for preventing bacterial wilt outbreaks.

Marker-assisted selection (MAS; Ashikari and Matsuoka 2006) is used to screen offspring for the wanted genotypes by applying genetically linked markers, which can greatly improve breeding efficiency by minimizing the effort and time required for phenotypic assessments. Linkage analysis and bulked segregate analysis (BSA; Michelmore et al. 1991) are two popular methods to identify quantitative trait loci (QTLs) associated with target traits. Linkage analysis is based on bi-parental populations, and the factors affecting the detection of QTLs include population size, marker density, logarithm of odds (LOD) threshold, phenotypic variance explained (PVE), and genetic distances between QTLs and markers ( $\mathrm{Li}$ et al. 2010). Bulked segregate analysis enables the rapid identification of markers linked to any specific gene or genomic region using two bulked from a segregating population with contrasting phenotypic traits (Michelmore et al. 1991). With the rapid development of sequencing technologies, high-throughput genotyping methods are increasingly applied to assist in high-resolution genetic mapping.

Some resistant QTLs for peanut bacterial wilt have been identified via linkage analysis and BSA. For example, two major QTLs on linkage groups LG1 and LG10, with PVE of $12-21 \%$, were detected using a linkage map constructed with simple sequence repeat (SSR) and single nucleotide polymorphism (SNP) markers (Zhao et al. 2016). A major and stable QTL for bacterial wilt resistance ( $q$ BWB02.1) was mapped on chromosome B02 using a high-density SNPbased genetic linkage map with a recombinant inbred line (RIL) population derived from the YZ9012 × Xuzhou68-4 cross (Wang et al. 2018). Luo et al. (2019) selected lines with contrasting phenotypes from the same RIL population and identified a QTL in the same linkage region by BSA. Moreover, two major QTLs were detected on chromosome B02 using linkage mapping and QTL-seq based on a RIL population derived from a cross between peanut cultivars Xuhua13 and Zhonghua6 (Luo et al. 2020). In conclusion, one major QTL on chromosome B02 was repeatedly detected using different methods and populations. Two diagnostic markers were successfully validated in the lines derived from Yuanza9102 but couldn't distinguish other susceptible and resistant cultivars (Luo et al. 2019).

A RIL population comprising 521 lines derived from the cross of Yuanza9102 $\times$ wt09-0023 was used to fine map the QTL for resistance to bacterial wilt of peanut via highthroughput sequencing. The objectives of this study were: (1) to construct a high-density genetic map according to the dRAD sequencing protocol; (2) to map the resistant QTL for peanut bacterial wilt; (3) to fine map the resistant QTL to a smaller marker interval; (4) to develop Kompetitive
Allele-Specific PCR (KASP) markers for MAS and validate their efficacy using different panels of peanut germplasms; and (5) to identify candidate genes for bacterial wilt resistance.

\section{Materials and methods}

\section{Plant materials}

A population comprising $521 \mathrm{~F}_{8: 10}$ RILs was derived from a cross between Yuanza9102 and wt09-0023 in 2010 at the experimental base of the Henan Academy of Agricultural Science, Yuanyang, China. The female and male parents were planted in two flowerpots, respectively, and three seeds were planted in each pot with $30 \mathrm{~cm}$ in high and $45 \mathrm{~cm}$ in diameter and then the SSD (single seed descent) method was used for generation acceleration. The female parent (Yuanza9102) is a widely grown variety with high and stable resistance to bacterial wilt and was released by the Institute of Industrial Crops, Henan Academy of Agricultural Science in 2002 (Luo et al. 2019). The male parent (wt09-0023) is a runner type peanut variety provided by Kim Moore (AgResearch Consultants Inc., ACI Seeds, USA) and highly susceptible line to bacterial wilt. The RILs were produced by planting offspring in Henan (in the summer season) and Hainan (in the winter season) for accelerating generation advancement through single seed descent. To validate the diagnostic markers, a panel consisting of 71 breeding lines, 47 of which were derived from the crosses involving the resistant parent (Yuanza9102), and another panel comprising 317 peanut germplasm lines were phenotyped and genotyped using the newly developed KASP markers. Additionally, peanut cultivars Zhonghua6 and Zhanghua12, which were released by the Oil Crops Research Institute of the Chinese Academy of Agricultural Sciences, were used as the resistance and susceptibility check varieties, respectively.

\section{dRAD library construction, sequencing, and SNP calling}

Leaf tissue of single $\mathrm{F}_{8}$ plants and the two parents were used for DNA isolation. The genomic DNA was first digested with restriction enzyme EcoRI, fragments of 300-500 bp were subsequently collected, amplified, and sequenced on an Illumina HiSeq 4000 platform to generate 150 -bp pairedend reads. The sequencing depths for the two parents and the RILs were approximately $25 \times$ and $5 \times$, respectively. After filtering the reads to eliminate adapters and low-quality reads, the remaining clean reads were aligned to the reference genome (Bertioli et al. 2019) using the bwa-0.7.10 (Li and Durbin 2019). The uniquely mapped reads were used to call SNPs with the SAMtools v0.1.19 and GATK v3.3.0 
programs (McKenna et al. 2010). The homozygous and polymorphic SNPs between the two parents were retained for the RIL population. The SNPs with missing and heterozygosity rates less than $10 \%$ and a sequencing depth greater than $3 \times$ were used for the following analysis. The clean data obtained in this study have been submitted to the BioProject database of NCBI (BioProject ID: PRJNA704994).

\section{Genetic map construction and QTL mapping}

The filtered SNPs of the $521 \mathrm{~F}_{8}$ lines were used to construct a genetic linkage map. First, the BIN module of QTL IciMapping v4.2 (Meng et al. 2015) was used to delete the completely linked, redundant markers. Next, Joinmap v5.0 (Van Ooijen 2018) was used to identify linkage groups (LGs) based on the independence LOD scores from 2 to 28 , and the maximum likelihood algorithm was applied for determining loci orders using Kosambi's mapping function. The genetic map was plotted using the R package LinkageMapView (Ouellette et al. 2018). QTL analysis was performed using multiple QTL mapping (MQM) of MapQTL v6.0 (Van Ooijen 2009), by setting the mapping step size as $0.1 \mathrm{cM}$ and the LOD threshold as 2.5 .

\section{Development of KASP markers for MAS and validation of the markers in broad germplasm collections}

The polymorphic SNPs between the two parents (wholegenome resequencing data; approximately $20 \times$ coverage) in the region covered by the putative QTL on LG12 including $1 \mathrm{Mb}$ up- and downstream were converted to KASP markers according Trick et al. (2012). Additionally, 521 RILs, 71 breeding lines, and 317 peanut germplasms were genotyped using the newly developed KASP markers. Phenotypes were generated for all the lines and the marker/trait-association validated to narrow down the candidate gene region. The QTL region of LG12 was drawn with the MapChart v2.3 (Voorrips 2002).

\section{Phenotyping of bacterial wilt resistance}

A disease nursery established in 1980 in Hezhou city, the Guangxi Zhuang Autonomous Region, China, was used to assess bacterial wilt resistance. Because of the limited size of the disease nursery field, the plant material was tested in consecutive trials from 2016 to 2020 . The parental lines and 412 lines of the RIL population were assessed in 2016 and 2017 ( $F_{9}$ and $F_{10}$ generations), the two parents and the remaining 109 lines of the RIL population $\left(\mathrm{F}_{10}\right.$ generation) and 71 breeding lines were assessed in 2018, and eventually, 317 peanut germplasms were assessed in 2019 and 2020 . Twenty seeds of each material were sown in a randomized block design with two replications, and the resistant and susceptible check varieties were included in each block. The number of emerged plants was recorded 10-16 days after sowing. Survival rates were calculated at the seedling (20-30 days after sowing), flowering (31-40 days), pegging (41-60 days), and harvesting (120-130 days) stages. The survival rate was calculated using the following formula: Survival rate $(\%)=($ no. of survived plants/no. of emerged plants) $\times 100$.

\section{Statistical analysis of phenotyping data}

An analysis of variance (ANOVA) among the 412 lines over their survival rates at each of the four different stages was performed in 2016 and 2017 using the AOV module of QTL IciMapping v4.2 (Meng et al. 2015), and broad-sense heritability was estimated accordingly. The distribution of survival rates was visualized in box, and violin plots produced using R v3.5.

\section{Results}

\section{Phenotypic variations of bacterial wilt resistance}

The female parent (Yuanza9102) and the male parent (wt090023) displayed clear difference in resistance to bacterial wilt at flowering, pegging, and harvesting stages across 3 years (Table S1). Additionally, considerable phenotypic variations in response to bacterial wilt were observed among the RILs at four observed stages across the 3 years (Table S1 and Fig. 1). The survival rates of a subset of 412 lines were investigated in 2016 and 2017, and that of all 521 lines were included in 2018, in which the average of the survival rates of the 412 lines recorded in 2016 and 2017 was used. The kurtosis and skewness values according Table $\mathrm{S} 1$ as well as the violin plots in Fig. 1D showed that the survival rates at the harvesting stage were closer to a normal distribution. The ANOVA results indicated a highly significant genotype effect $(G)$ at all four stages, but the environment $(E)$ and genotype $\times$ environment interactions $(G \times E)$ were less significant or not significant (Table S2), the reason may be that the experiment was conducted in a single location. The broad-sense heritability ranged from 0.8 to 0.9 based on entry mean at four stages (Table S2).

\section{dRAD sequencing and SNP identification}

The dRAD sequencing protocol was used for analyzing the two parents and 521 RILs. Approximately $1918 \mathrm{~Gb}$ clean data (Q20 > 94\%) were generated, including 13.4 billion reads (Table S3). About 116.3 and 132.8 million reads 

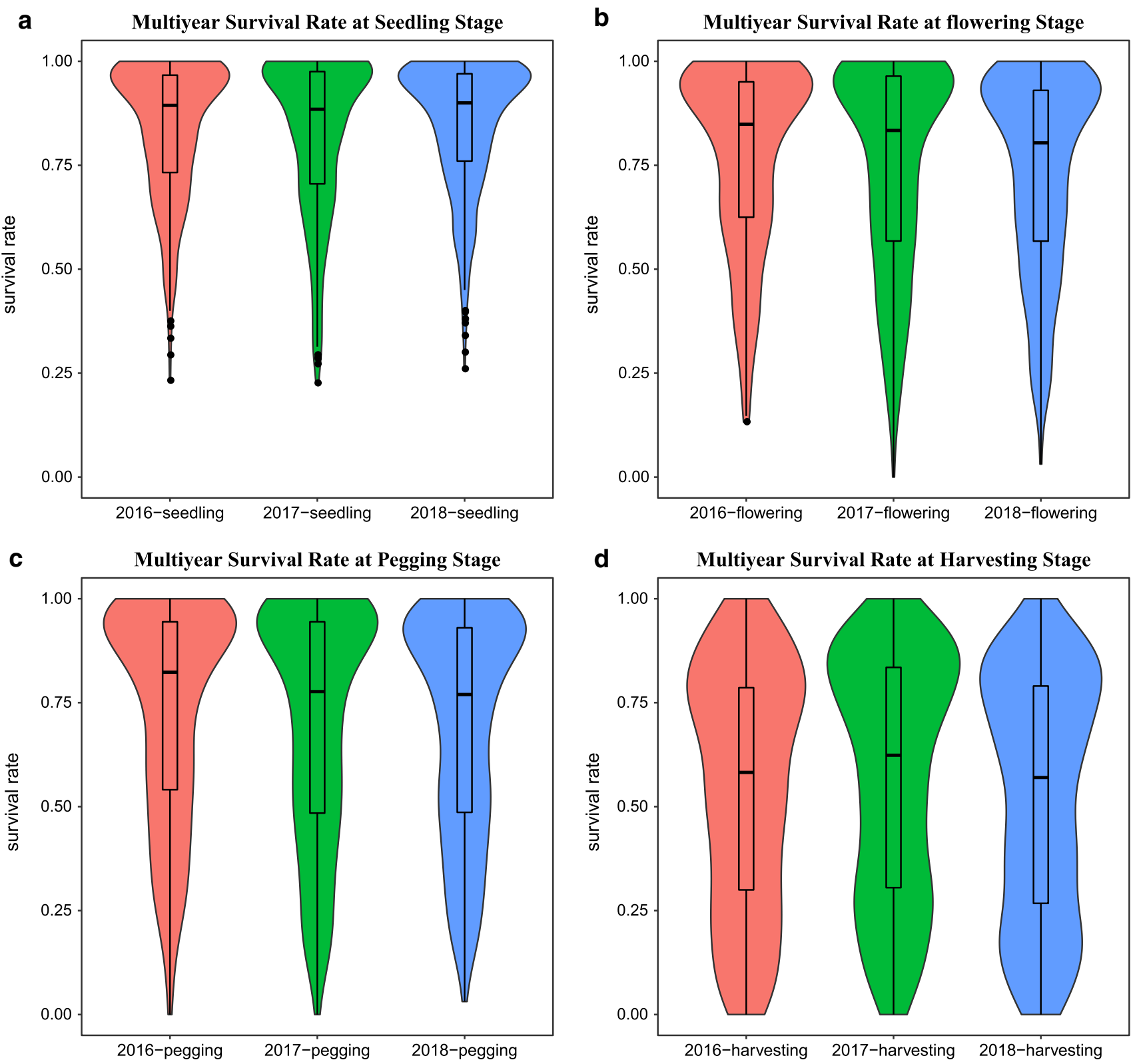

Fig. 1 Boxplots and violin plots for the survival rate of RILs at four stages across 3 years. A seedling stage; $\mathbf{B}$ flowering stage; $\mathbf{C}$ pegging stage; $\mathbf{D}$ harvesting stage. The survival rate of 412 lines was assessed

in 2016 and 2017, and that of all 521 lines was contained in 2018, in which for the survival rate of the 412 lines, the average of the survival rate in 2016 and 2017 was used

were obtained for the female and male parents, respectively, whereas 10.5-62.1 million reads were obtained for the RILs (Table S3). More than $98 \%$ of the reads were mapped to the reference genome (Table S3). A total of 291,195 polymorphic SNPs were identified for the two parents, of which 90,807 were homologous and used to screen for SNPs in the RILs. The resulting 29,301 SNPs were further filtered, after which the 24,852 remaining SNPs were used for the following analysis.

\section{Construction of the genetic linkage map}

Redundant markers were removed from the filtered SNP set using the BIN module of QTL IciMapping v4.2, and the remaining 5151 SNPs were used to construct a genetic linkage map based on the 521 lines using Joinmap v5.0. After eliminating 31 redundant SNPs, 5120 SNPs were assigned to 20 LGs (Table 1 and Fig. S1). The whole map length was about $3179 \mathrm{cM}$, with an average marker distance of $0.6 \mathrm{cM}$ 
Table 1 Summary of the highdensity linkage groups (LGs) obtained for the RIL population

\begin{tabular}{|c|c|c|c|c|c|}
\hline Linkage ID & Total marker & $\begin{array}{l}\text { Total dis- } \\
\text { tance }(\mathrm{cM})\end{array}$ & $\begin{array}{l}\text { Average dis- } \\
\text { tance }(\mathrm{cM})\end{array}$ & $\begin{array}{l}\text { Max inter-marker } \\
\text { distance }(\mathrm{cM})\end{array}$ & $\begin{array}{l}\text { Inter-markers } \\
\text { distances } \leq 3 \mathrm{cM} \\
(\%)\end{array}$ \\
\hline LG01 & 228 & 133.8 & 0.6 & 4.6 & 98.7 \\
\hline LG02 & 284 & 170.7 & 0.6 & 16.5 & 98.2 \\
\hline LG03 & 255 & 175.6 & 0.7 & 20.1 & 97.7 \\
\hline LG04 & 158 & 100.0 & 0.6 & 9.9 & 96.8 \\
\hline LG05 & 291 & 162.3 & 0.6 & 9.1 & 99.0 \\
\hline LG06 & 276 & 169.6 & 0.6 & 8.9 & 99.3 \\
\hline LG07 & 203 & 151.2 & 0.7 & 17.0 & 94.6 \\
\hline LG08 & 221 & 144.6 & 0.7 & 9.8 & 95.9 \\
\hline LG09 & 321 & 192.1 & 0.6 & 4.3 & 98.1 \\
\hline LG10 & 114 & 101.1 & 0.9 & 10.8 & 93.0 \\
\hline LG11 & 322 & 159.1 & 0.5 & 7.4 & 98.5 \\
\hline LG12 & 349 & 191.7 & 0.6 & 3.8 & 98.6 \\
\hline LG13 & 209 & 167.7 & 0.8 & 12.6 & 96.7 \\
\hline LG14 & 374 & 188.7 & 0.5 & 7.6 & 98.9 \\
\hline LG15 & 479 & 211.3 & 0.4 & 4.0 & 99.6 \\
\hline LG16 & 210 & 139.4 & 0.7 & 5.5 & 97.1 \\
\hline LG17 & 118 & 168.7 & 1.4 & 21.9 & 89.8 \\
\hline LG18 & 154 & 126.5 & 0.8 & 17.2 & 93.5 \\
\hline LG19 & 115 & 121.2 & 1.1 & 25.8 & 93.9 \\
\hline LG20 & 439 & 204.1 & 0.5 & 5.2 & 98.4 \\
\hline Total & 5120 & 3179.4 & 0.6 & - & 96.8 \\
\hline
\end{tabular}

(Table 1). The marker number among linkage groups varied from 114 (LG10) to 479 (LG15), the map length ranged from $100.0 \mathrm{cM}$ (LG04) to $211.3 \mathrm{cM}$ (LG15), and the average marker distance ranged from 0.4 (LG15) to $1.1 \mathrm{cM}$ (LG19) (Table 1). The maximum inter-marker distance was $25.8 \mathrm{cM}$ (LG19), and $96.8 \%$ of the inter-marker distances were less than $3.0 \mathrm{cM}$. The collinearity analysis revealed a high degree of synteny between the genetic linkage map and the corresponding chromosomes of the reference genome, with the exception of some translocations between LG03 and LG13 and between LG06 and LG16 (Fig. 2).

\section{QTL identification and fine mapping for bacterial wilt resistance}

QTL identification for bacterial wilt resistance was performed, respectively, based on survival rates of the 412 RILs at four development stages in 2016 and 2017. In 2018, the average survival rates for the 412 RILs based on the above mentioned 2 years' data together with the survival rates of the additional 109 lines collected in 2018 were used to identify QTLs. Four QTLs were detected in all 3 years, which were distributed on LG03, 12, 14, and 20, with an LOD score greater than 2.5 (Table 2). A major QTL on LG12 ( $q B W A 12)$ was consistently detected at all four stages in all 3 years, with LOD scores of 32.8-66.0, and with PVE of
31.2-44.8\% (Table 2). The additive effect was between 7.9 and 14.1, implying the resistance allele was derived from the female parent Yuanza9102. Moreover, $q B W A 12$ was located on chromosome 12 and was localized to a $2.7 \mathrm{~cm}$ interval (29.1-31.8 cM) flanked by SNP site A12.4210453 and A12.4601283, corresponding to a $390.8 \mathrm{~kb}$ physical region. To fine map $q B W A 12,42$ homologous and polymorphic SNPs between the two parents in a 3.3-5.1 Mb physical region of chromosome 12 were selected to convert to KASP markers. Then 17 successfully genotyped KASP markers (Table S4) confirmed polymorphisms between the two parents and were used to genotype the 521 RILs. These markers were integrated in the genetic map of LG12, and a fine mapping QTL analysis for bacterial wilt resistance was performed (Fig. 3). Accordingly, qBWA12 was fine mapped to a 0.6-cM-interval flanked by A12.4255141 and A12.4471816, and the physical region was shortened to $216.7 \mathrm{~kb}$ from $390.8 \mathrm{~kb}$.

\section{KASP marker validation}

A total of 71 breeding lines in 2018 (Table S5) and 317 peanut germplasms in 2019 and 2020 (Table S6) were evaluated for bacterial resistance. The 17 KASP markers successfully genotyped in the RIL population were used to genotype the two panels of peanut material (Figs. 4 and S2-4). For the 47 
Fig. 2 Collinearity of the linkage groups constructed using a RIL population with the reference genome (Arachis hypogaea.cv.tifrunner). The prefix LG stands for genetic map on left-hand side whereas, prefix chr stands for corresponding physical map on right-hand side

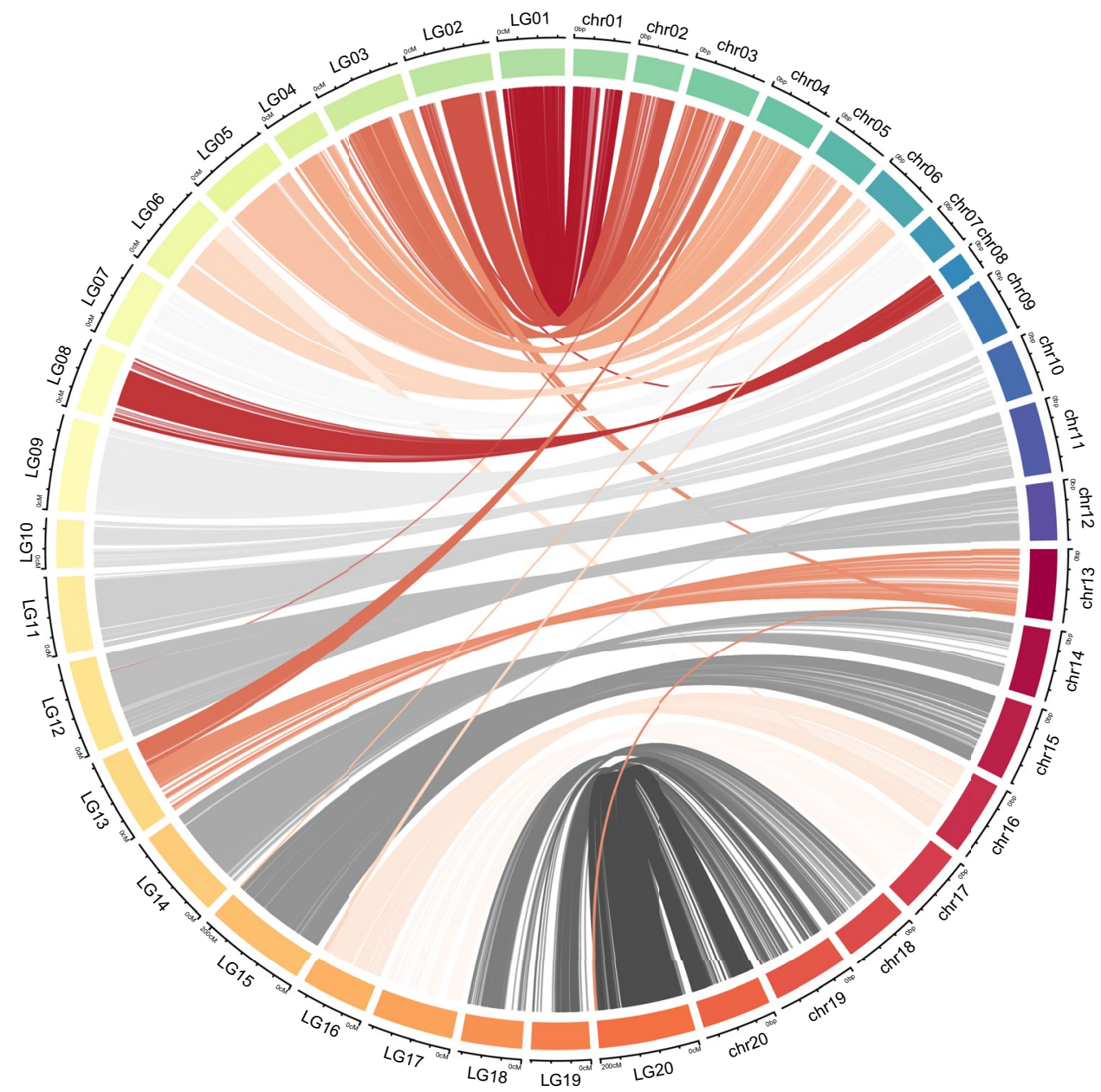

Table 2 QTLs identified for BW resistance at four stages in 3 years

\begin{tabular}{|c|c|c|c|c|c|c|c|}
\hline QTL & LG & Position (cM) & Left-right marker & LOD & $\operatorname{PVE}(\%)$ & Add effects $(\%)$ & Environment \\
\hline$q B W A 03$ & LG03 & $101.7-102.4$ & A03.128299006-A03.128522169 & $2.6-5.7$ & $2.5-4.9$ & $2.9-4.8$ & $\begin{array}{l}\text { (2016_harvesting; 2017_fruiting; } \\
\text { 2017_harvesting; 2018_flower- } \\
\text { ing; 2018_fruiting; 2018_har- } \\
\text { vesting) }\end{array}$ \\
\hline$q B W A 12$ & LG12 & $29.1-31.8$ & A12.4210453-A12.4601283 & $32.8-66.0$ & $31.2-44.8$ & $7.9-14.1$ & $\begin{array}{l}\text { (2016_seedling; 2016_flowering; } \\
\text { 2016_fruiting; 2016_harvest- } \\
\text { ing; 2017_seedling; 2017_flow- } \\
\text { ering; 2017_fruiting; 2017_- } \\
\text { harvesting; 2018_seedling; } \\
\text { 2018_flowering; 2018_fruiting; } \\
\text { 2018_harvesting) }\end{array}$ \\
\hline$q B W A 14$ & LG14 & $59.4-60.0$ & A14.9011103-A14.9111536 & $3.0-4.9$ & $3.2-5.3$ & -4.8 to -3.9 & $\begin{array}{l}\text { (2016_harvesting; 2017_fruiting; } \\
\text { 2017_harvesting; 2018_harvest- } \\
\text { ing) }\end{array}$ \\
\hline$q B W A 20$ & LG20 & $182.6-182.8$ & A20.141889915-A20.141884654 & $2.5-4.4$ & $2.2-5.0$ & $2.0-4.6$ & $\begin{array}{l}\text { (2016_seeding; 2016_flowering; } \\
\text { 2016_harvesting; 2017_seed- } \\
\text { ling; 2017_flowering; 2017_- } \\
\text { fruiting; 2017_harvesting; } \\
\text { 2018_seedling; 2018_flower- } \\
\text { ing; 2018_fruiting; 2018_har- } \\
\text { vesting) }\end{array}$ \\
\hline
\end{tabular}


LG12

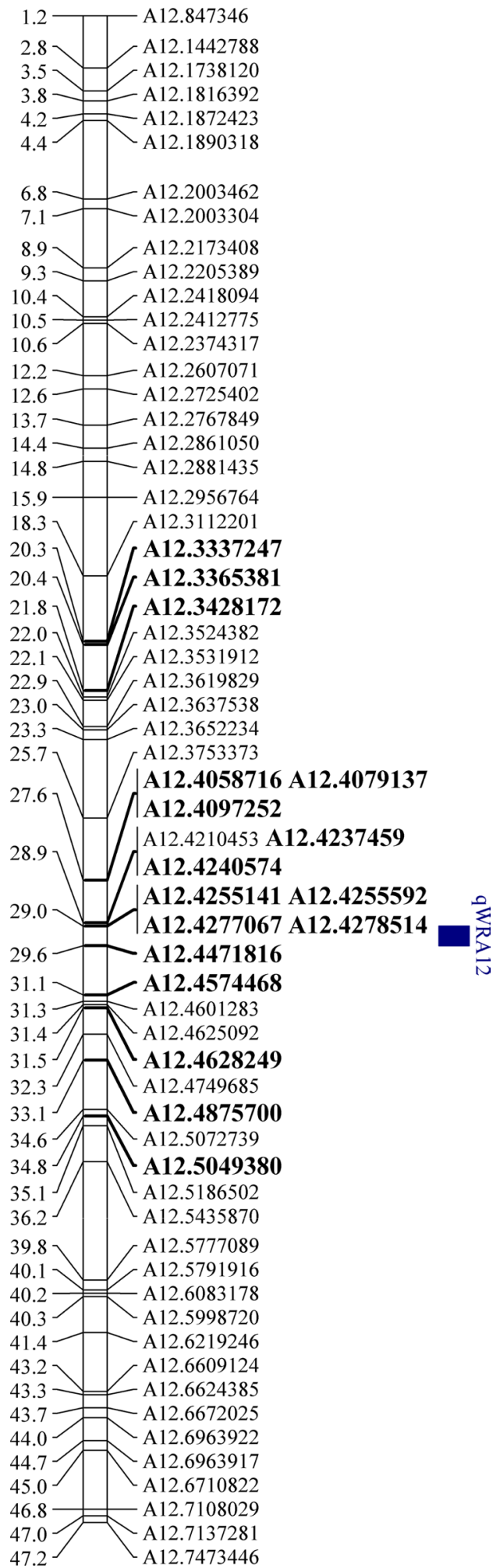

2017

2018

LOD

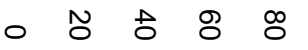

LOD

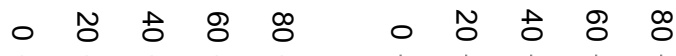
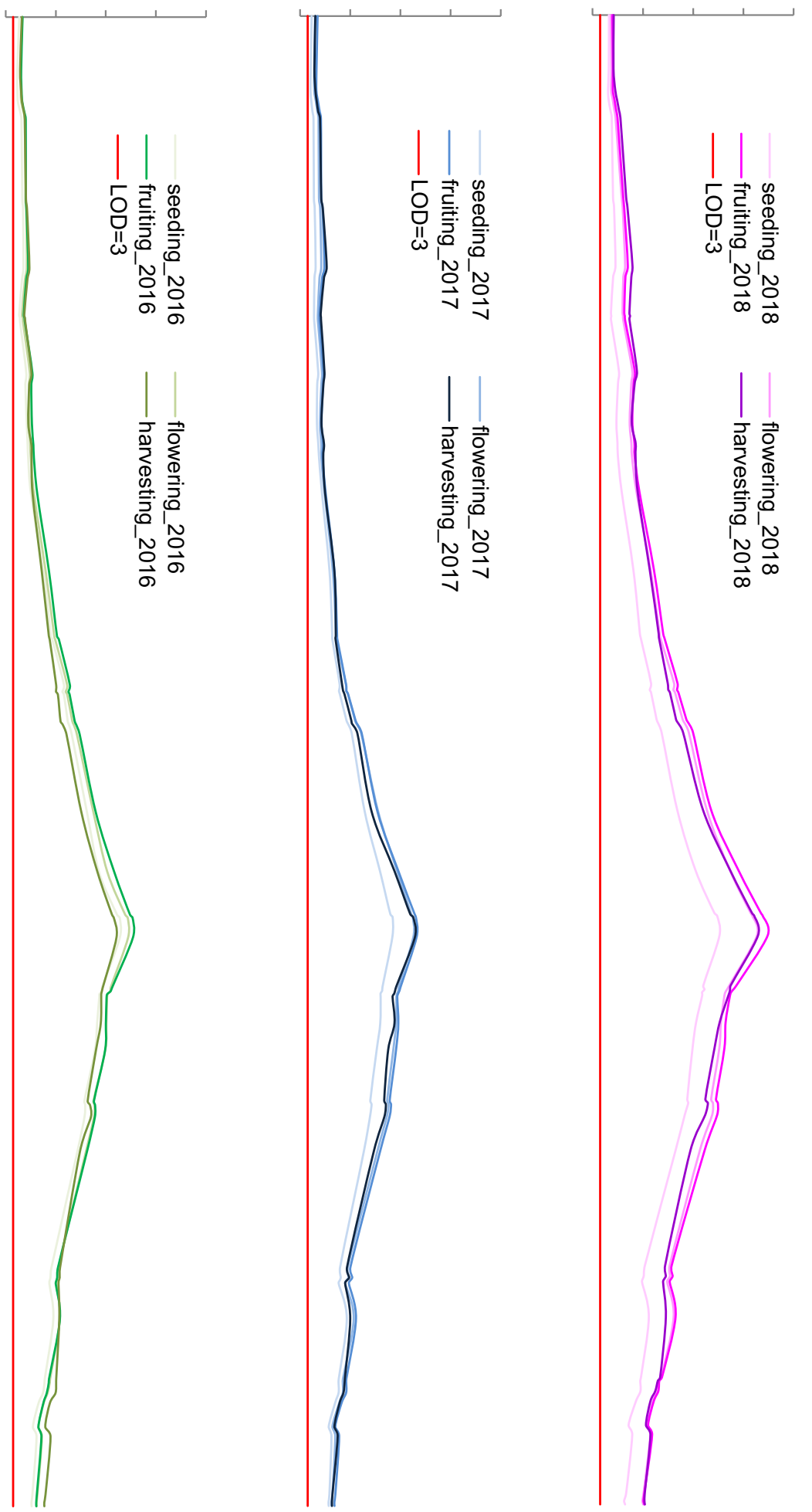

Fig. 3 LOD curves and position of the $q B W A 12$ on LG12 under four stages in 3 years. Only the linkage area close to $q B W A 12$ is shown 
Fig. 4 Phenotypic differences between two genotypes at three SNP sites for 71 breeding lines and 317 peanut germplasms lines. a Phenotypic differences between two genotypes at three SNP sites for 47 lines derived from Yuanza9102. b Phenotypic differences between two genotypes at three SNP sites for 24 breeding lines. c Phenotypic differences between two genotypes at three SNP sites for 317 peanut germplasms
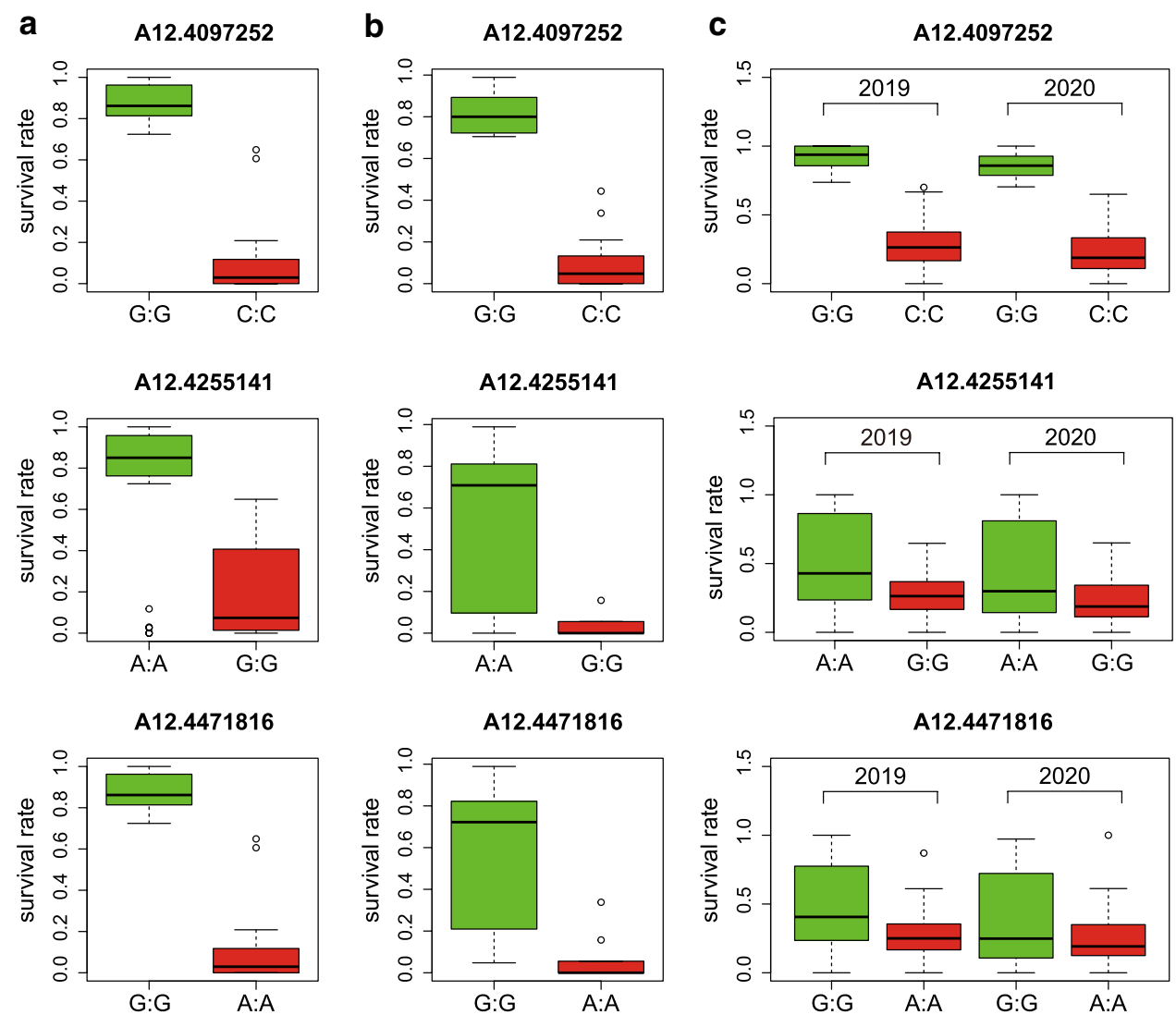

breeding lines derived from Yuanza9102, significant phenotypic differences were observed between the two genotypes at 14 SNP sites (A12.4058716-A12.5049380) (Fig. S2), including A12.4097252 and the two flanking markers A12.4255141 and A12.4471816 (Fig. 4a). In contrast, for the 24 breeding lines that were not derived from Yuanza9102 as well as the 317 peanut germplasms, significant phenotypic differences were observed between the two genotypes only at A12.4097252 (Fig. 4b, c, Figs. S3-4). These results implicated that A12.4097252 was tightly linked with bacterial wilt resistance, and the resistant allele was $\mathrm{G}$ instead of $\mathrm{C}$ at the SNP site.

\section{Potential candidate genes for bacterial wilt resistance}

Nineteen candidate genes for bacterial wilt resistance were predicted in the region covered by $q B W A 12$ on the basis of the annotated reference genome database (Bertioli et al. 2019) (Table 3). Eleven of these candidate genes were nucleotide-binding site-leucine-rich repeat (NBS-LRR) genes, and one gene (arahy.KAG5PZ) encoded a disease resistance-related protein with the NB-ARC (nucleotidebinding adapter shared by APAF-1, R proteins, and CED-4) domain along with the LRR (Table 3). To further screen the candidate genes, the whole-genome resequencing reads of
Yuanza9102 were aligned to the reference genome, and the mapping rates in the regions covered by the 19 candidate genes were determined (Table 3). Nine genes, with mapping rates of $100 \%$, may be unrelated to bacterial wilt resistance, including two NBS-LRR genes and the NB-ARC-LRR gene. Another nine NBS-LRR genes, with mapping rates of 38.0-99.9\%, may play an important role in resistance to bacterial wilt. The alignment of reads to the eight genes with a mapping rate less than $96.0 \%$ visualized using the Integrative Genomics Viewer (Robinson et al. 2011) that is presented in Figure S5.

\section{Discussion}

Cultivated peanut (Arachis hypogaea $\mathrm{L}$.) is an allotetraploid species (AABB, $2 n=4 x=40$ ) with a large genome (approximately $2.7 \mathrm{~Gb}$ ). It diverged from two diploid ancestral species about 9400 years ago, which resulted in limited genetic diversity (Bertioli et al. 2016). Therefore, the first two generations of molecular markers were not widely used for cultivated peanut (Pandey et al. 2014; Chen et al. 2016). The advance of next-generation sequencing technology and the publication of peanut genomes (Bertioli et al. 2016, 2019; 
Table 3 The predicted candidate genes for BW resistance in the region covered by the major $q B W A 12$

\begin{tabular}{|c|c|c|c|c|c|}
\hline ID & gene model & $\mathrm{chr}$ & Gene location & annotation & Mapping rate $(\%)$ \\
\hline 1 & arahy.AU62Q4 & A12 & $4209114-4210556(-)$ & UDP-Glycosyltransferase superfamily protein & 100.0 \\
\hline 2 & arahy. V6I7WA & A12 & $4236131-4239737(+)$ & Disease resistance protein (NBS-LRR class) & 95.2 \\
\hline 3 & arahy. $45 D 7 Q Y$ & A12 & $4242720-4243363(-)$ & Pre-mRNA-splicing factor cwc 26 & 100.0 \\
\hline 4 & arahy.TE7IIT & A12 & $4257212-4259902(+)$ & disease resistance Protein (NBS-LRR class) & 99.9 \\
\hline 5 & arahy. $0 E H V 1 A$ & A12 & $4280257-4284123(+)$ & Disease resistance protein (NBS-LRR class) & 93.6 \\
\hline 6 & arahy. $K A G 5 P Z$ & A12 & $4292471-4292740(+)$ & LRR and NB-ARC domain disease resistance protein & 100.0 \\
\hline 7 & arahy. W2W4JI & A12 & $4292896-4294501(+)$ & Disease resistance protein (NBS-LRR class) & 100.0 \\
\hline 8 & arahy.P88NCS & A12 & $4323774-4327025(+)$ & Disease resistance protein (NBS-LRR class) & 91.6 \\
\hline 9 & arahy.HZH8EM & A12 & $4339150-4343716(+)$ & Disease resistance protein (NBS-LRR class) & 52.6 \\
\hline 10 & arahy.5D95TJ & A12 & $4352358-4356221(+)$ & Disease resistance protein (NBS-LRR class) & 89.8 \\
\hline 11 & arahy.D89ZXX & A12 & $4365013-4366609(+)$ & Disease resistance protein (NBS-LRR class) & 100.0 \\
\hline 12 & arahy.WQJN9J & A12 & $4394900-4398148(+)$ & Disease resistance protein (NBS-LRR class) & 80.0 \\
\hline 13 & arahy. LURW66 & A12 & $4410277-4414843(+)$ & Disease resistance protein (NBS-LRR class) & 38.0 \\
\hline 14 & arahy.MXY2PU & A12 & $4423482-4426655(+)$ & Disease resistance protein (NBS-LRR class) & 86.0 \\
\hline 15 & arahy. TC $7 Y O P$ & A12 & 4442239-4445553(-) & WRKY family transcription factor & 100.0 \\
\hline 16 & arahy. $K L 1 Z 6 U$ & A12 & $4458290-4461891(-)$ & Histone superfamily protein & 100.0 \\
\hline 17 & arahy.G8BSRK & A12 & $4463005-4471216(-)$ & Alpha/beta hydrolase domain-containing protein 11 & 100.0 \\
\hline 18 & arahy.DSQC6N & A12 & $4474755-4474964(-)$ & Uncharacterized protein & 100.0 \\
\hline 19 & arahy.GPD66T & A12 & $4479488-4482537(+)$ & Pentatricopeptide (PPR) repeat-containing protein & 94.2 \\
\hline
\end{tabular}

Chen et al. 2019; Zhuang et al. 2019) have accelerated QTL mapping-related research and MAS-based breeding.

\section{Improved genetic linkage map construction and QTL mapping}

The publication of the first peanut reference genome (Bertioli et al. 2016) and the development of second generation sequencing methods greatly increased the efficiency and accuracy of QTL mapping in peanut (Shasidhar et al. 2017; Wang et al. 2018; Han et al. 2018). The first genetic linkage maps were mainly constructed using SSR markers, and these maps were limited to a few hundreds of markers (Huang et al. 2015; Prasad et al. 2015; Tseng et al. 2016; Zhao et al. 2016). Today, most genetic maps are constructed using SNP markers from next generation sequencing, and often, over 2000 SNPs, which are ten times more than formerly used SSR markers (Wang et al. 2018; Gangurde et al. 2020; Li et al. 2019; Liu et al 2020; Zhang et al. 2019), are mapped. Additionally, using the A. hypogaea genome as a reference genome substantially increased the mapping rate of sequencing data as well as the number of SNPs. Luo et al. (2019) obtained 122 million homologous and polymorphic SNPs between two parents using the genomes of A. duranensis and A. ipaensis as reference while 326 million SNPs were called using the genome of A. hypogaea $\mathrm{cv}$. Tifrunner as reference with the same sequencing protocol and similar sequencing depth (unpublished data).
In addition to marker density, population size is also one critical factor for resolution and power of QTL mapping ( $\mathrm{Li}$ et al. 2010). The two RIL populations included in studies by Wang et al. (2018) and Luo et al. (2020) comprised 188 and 268 lines, respectively, and the final physical regions covered by the resistant QTL for peanut bacterial wilt were both $\sim 2 \mathrm{Mb}$. In the current study, the physical interval was narrowed down to about $390.8 \mathrm{~kb}$ using 521 lines. Because a larger population size results in more recombinant events, smaller QTL confidence intervals and stronger linkage between QTL and phenotypes can be achieved.

\section{Collinearity analysis of the genetic linkage and physical maps}

An analysis of the LGs and their corresponding chromosomes revealed a relatively high collinearity between the LGs and chromosomes, with the exception of four LGs (Fig. 2). The marker orders in some regions on LG03, LG06, LG13, and LG16 were inconsistent with the corresponding physical orders (Fig. 2) because two reciprocal translocations occurred between the ends of LG03 and LG13 and between the ends of LG06 and LG16. For details, the SNPs from Arahy.13:135672462-145340054 were mapped to the end of LG03, and the SNPs from Arahy.03:133160681-141868592 were grouped at the end of LG13. Similarly, the SNPs from Arahy.16:143810297-150964456 and Arahy.06:108333059-111980823 were mapped at 
the ends of LG06 and LG16, respectively. There were also some translocations between LG02 and Arahy.08 and between LG20 and Arahy.13. The SNPs from Arahy.08:51599619-51799542 were mapped to the head of LG02, and the SNPs from Arahy.13:145768538-146301976 were mapped to the end of LG20. These translocations resulted from assembly mistakes in the reference genome (A. hypogaea cv. Tifrunner version 1). A large portion of the distal end was swapped between Arahy.03 and Arahy.13 and between Arahy.06 and Arahy.16 in the updated genome ( $A$. hypogaea $\mathrm{cv}$. Tifrunner version 2 ; https://www.peanutbase. org/peanut_genome_v1_v2). Hence, the genetic map may provide valuable information for the assembled genome.

\section{Comparison between linkage mapping and BSA}

Linkage mapping and BSA are two widely used methods for identifying QTLs associated with target traits, but their mapping power and resolution are not comparable. For the same RIL population (Yuanza9102 $\times$ Xuzhou68-4), a physical interval of approximately $2.3 \mathrm{Mb}$ (chromosome B02:2501128-4797039) covered by $q B W B 02.1$ was obtained from linkage mapping (Wang et al. 2018), whereas a larger interval (approximately $3.4 \mathrm{Mb}$ ) was obtained using the BSA method (Luo et al. 2019). Thus, the mapping resolution of linkage analysis is greater than that of BSA, especially for QTLs with a small PVE. Although, sequencing costs were greatly reduced for BSA, only two bulked pools consisting of a few individuals with extreme phenotypes were sequenced, but the identified QTL had a larger interval, and more makers and recombinants would be needed to fine-map the QTL. In the present study, the identification of QTL $q B W A 12$ for peanut bacterial wilt spanned a physical region of only $390.8 \mathrm{~kb}$ once again demonstrated the high resolution of extensive RIL linkage mapping.

\section{Predicted candidate genes}

Proteins containing NBS-LRRs, which exist in many plants, form the biggest group of $\mathrm{R}$ proteins involved in effectortriggered immunity (Jones and Dangl 2006). These proteins can enhance plant resistance to various pathogens. For example, two NBS-LRR genes (RPS4 and RRS1) cloned from Arabidopsis thaliana reportedly mediate resistance to several pathogens (e.g., bacterial wilt pathogen) in other transgenic plants, including oilseed rape, tomato, and cucumber (Gassmann et al.1999; Deslandes et al. 2002; Vinatzer et al. 2013; Narusaka et al. 2014). Moreover, the expression of a novel peanut NBS-LRR gene (AhRRS5) is up-regulated in both resistant and susceptible peanut cultivars in response to $R$. solanacearum, and the overexpression of this gene enhances the resistance of transgenic tobacco plants to $R$. solanacearum (Zhang et al. 2017).
Using the annotated Tifrunner reference genome (Bertioli et al. 2019), 11 NBS-LRR and one NB-ARC-LRR genes were detected in the interval covered by $q B W A 12$ (Table 3). The clean resequencing reads for the resistant parent (Yuanza9102) were precisely mapped to two NBSLRR and the NB-ARC-LRR genes, with a mapping rate of $100 \%$, whereas the mapping rates were $38.0-99.9 \%$ for nine other NBS-LRR genes. The relatively low mapping rates may be explained by the considerable differences in the gene sequences between Yuanza9102 and Tifrunner. Furthermore, the possible contributions of these nine NBS-LRR genes to the resistance of peanut to bactrial wilt will need to be investigated.

\section{KASP marker design and validation}

Two KASP markers (AraipB02:3740746 and AraipB02:5804063) were designed and validated using the RIL population derived from the Yuanza9102 $\times$ Xuzhou68-4 cross (Luo et al. 2019). The data revealed that both markers can only be used for the lines derived from Yuanza9102 and conclude that the resistance allele from Yuanza9102 may differ from the resistance alleles in other resistant lines (Luo et al. 2019). In this study, 17 polymorphic SNPs between the two parents in the target QTL interval, which were detected by analyzing the whole-genome resequencing data, were used to develop KASP markers for fine mapping $q B W A 12$ and validated using two panels of peanut materials. Among of them, 14 KASP markers, including two markers flanking qBWA12 (A12.4255141 and A12.4471816), can be used to screen for resistant lines derived from Yuanza9102, while only one (A12.4097252) is applicable for all peanut germplasms. Therefore, A12.4097252 can be applied in markerassisted breeding to develop peanut varieties resistant to bacterial wilt with various genetic backgrounds, while the other 13 flanking markers could be employed in the screening of the resistant genotypes derived from Yuanza9102.

Supplementary Information The online version contains supplementary material available at https://doi.org/10.1007/s00122-022-04033-y.

Acknowledgements The authors thank Dr. Georg Kurt Koch for fruitful discussions of QTL analysis and for editing the English text of the manuscript and thank Liwen Bianji (Edanz) (www.liwenbianji.cn/) for editing the English text of this manuscript. This work was supported by the National Basic Research Program of China, Special Project for National Supercomputing Zhengzhou Center Innovation Ecosystem Construction (201400210600), Major Science and Technology Projects of Henan Province (201300111000), China Agriculture Research System (CARS-13), Henan Provincial Agriculture Research System, China (S2012-5), Science and Technology Project of Henan Province (212102110254), Henan Provincial Young Talents Supporting Project (2020HYTP044), and Outstanding Young Scientists of Henan Academy of Agricultural Sciences (2020YQ08). 
Author contributions statement FQ performed laboratory experiments and genotype analysis. ZS performed phenotypic analysis and wrote the manuscript. HL and LQ developed the RIL population. QC, HL, and XL provided help in field experiments. LS, LM, MT, and XW provided help in laboratory and field experiments. $\mathrm{ZZ}, \mathrm{BH}$, and WD provided help in designing the experiments. XZ conceived and designed the experiments, facilitated the project, and assisted in manuscript preparation. All authors read and approved the final manuscript.

Funding This work was supported by the Special Project for National Supercomputing Zhengzhou Center Innovation Ecosystem Construction (201400210600), Major Science and Technology Projects of Henan Province (201300111000), China Agriculture Research System (CARS-13), Henan Provincial Agriculture Research System, China (S2012-5), Science and Technology Project of Henan Province (212102110254), Henan Provincial Young Talents Supporting Project (2020HYTP044), Outstanding Young Scientists of Henan Academy of Agricultural Sciences (2020YQ08), and the Henan Provicial R\&D Projects of Inter-regional Cooperation for Local Scientific and Technological Development Guided by Central Government (YDZX20214100004191).

Data availability All data generated or analyzed during this study are included in the manuscript and its Additional file 1 to Additional file 9. The clean resequencing data obtained in this study are available at the BioProject database at NCBI under the BioProject ID: PRJNA704994 (https://dataview.ncbi.nlm.nih.gov/object/PRJNA704994?reviewer= hsjf26260jisd87vn2p091s5u6). Materials used in this study are available from the corresponding authors.

\section{Declarations}

Conflict of interest Authors declare that there are no conflicts of interest.

Ethical approval Not applicable.

Open Access This article is licensed under a Creative Commons Attribution 4.0 International License, which permits use, sharing, adaptation, distribution and reproduction in any medium or format, as long as you give appropriate credit to the original author(s) and the source, provide a link to the Creative Commons licence, and indicate if changes were made. The images or other third party material in this article are included in the article's Creative Commons licence, unless indicated otherwise in a credit line to the material. If material is not included in the article's Creative Commons licence and your intended use is not permitted by statutory regulation or exceeds the permitted use, you will need to obtain permission directly from the copyright holder. To view a copy of this licence, visit http://creativecommons.org/licenses/by/4.0/.

\section{References}

Afroz A, Khan MR, Ahsan N, Komatsu S (2009) Comparative proteomic analysis of bacterial wilt susceptible and resistant tomato cultivars. Peptides 30(9):1600-1607. https://doi.org/10.1016/j. peptides.2009.06.005

Ashikari M, Matsuoka M (2006) Identification, isolation and pyramiding of quantitative trait loci for rice breeding. Trends Plant Sci 11:344-350
Bertioli DJ, Cannon SB, Froenicke L, Huang G, Farmer AD, Cannon EKS et al (2016) The genome sequences of Arachis duranensis and Arachis ipaensis, the diploid ancestors of cultivated peanut. Nat Genet 48:438-446

Bertioli DJ, Jenkins J, Clevenger J, Dudchenko O, Gao D, Seijo G et al (2019) The genome sequence of segmental allotetraploid peanut Arachis hypogaea. Nat Genet 51:877-884

Chen W, Jiao Y, Cheng L, Huang L, Liao B, Tang M et al (2016) Quantitative trait locus analysis for pod- and kernel-related traits in the cultivated peanut (Arachis hypogaea L.). BMC Genet. https://doi. org/10.1186/s12863-016-0337-x

Chen X, Lu Q, Liu H, Zhang J, Hong Y, Lan H et al (2019) Sequencing of cultivated peanut, Arachis hypogaea, yields insights into genome evolution and oil improvement. Mol Plant 12:920-934

Deslandes L, Olivier J, Theulieres F, Hirsch J, Feng DX, Bittner-Eddy $P$ et al (2002) Resistance to Ralstonia solanacearum in Arabidopsis thaliana is conferred by the recessive RRS1-R gene, a member of a novel family of resistance genes. PNAS 99:2404-2409

Gangurde SS, Wang H, Yaduru S, Pandey MK, Fountain JC, Chu Y et al (2020) Nested-association mapping (NAM)-based genetic dissection uncovers candidate genes for seed and pod weights in peanut (Arachis hypogaea ). Plant Biotechnol J 18:1457-1471

Gassmann W, Hinsch EM, Brian SJ (1999) The Arabidopsis RPS4 bacterial-resistance gene is a member of the TIR-NBS-LRR family of disease-resistance genes. Plant J 3:265-277

Han SY, Yuan M, Clevenger JP, Li C, Hagan A, Zhang XY et al (2018) A SNP-based linkage map revealed QTL for resistance to early and late leaf spot diseases in peanut (Arachis hypogaea L.). Front Plant Sci 9:1012

Huang L, He H, Chen W, Ren X, Chen Y, Zhou X et al (2015) Quantitative trait locus analysis of agronomic and quality-related traits in cultivated peanut (Arachis hypogaea L.). Theor Appl Genet 128:1103-1115

Jiang G, Wei Z, Xu J, Chen H, Zhang Y, She X et al (2017) Bacterial wilt in China: history, current status, and future perspectives. Front Plant Sci 8:1549

Jones JD, Dangl JL (2006) The plant immune system. Nature 444:323-329

Lebeau A, Gouy M, Daunay MC, Wicker E, Chiroleu F, Prior P et al (2012) Genetic mapping of a major dominant gene for resistance to Ralstonia solanacearum in eggplant. Theor Appl Genet 126:143-158

Li H, Durbin R (2009) Fast and accurate short read alignment with Burrows-Wheeler transform. Bioinformatics 25:1754-1760

Li H, Hearne S, Bänziger M, Li Z, Wang J (2010) Statistical properties of QTL linkage mapping in biparental genetic populations. Heredity 105:257-267

Li L, Yang X, Cui S, Meng X, Mu G, Hou M et al (2019) Construction of high-density genetic map and mapping quantitative trait loci for growth habit-related traits of peanut (Arachis hypogaea L.). Front Plant Sci 10:745

Liu N, Guo J, Zhou X, Wu B, Huang L, Luo H et al (2020) Highresolution mapping of a major and consensus quantitative trait locus for oil content to a $\sim 0.8-\mathrm{Mb}$ region on chromosome A08 in peanut (Arachis hypogaea L.). Theor Appl Genet 133:37-49

Luo H, Pandey MK, Khan AW, Wu B, Guo J, Ren X et al (2019) Nextgeneration sequencing identified genomic region and diagnostic markers for resistance to bacterial wilt on chromosome B02 in peanut ( Arachis hypogaea L.). Plant Biotechnol J 17:2356-2369

Luo H, Pandey MK, Zhi Y, Zhang H, Xu S, Guo J et al (2020) Discovery of two novel and adjacent QTLs on chromosome B02 controlling resistance against bacterial wilt in peanut variety Zhonghua 6. Theor Appl Genet 133:1133-1148 
Mansfield J, Genin S, Magori S, Citovsky V, Sriariyanum M, Ronald $P$ et al (2012) Top 10 plant pathogenic bacteria in molecular plant pathology. Mol Plant Pathol 13:614-629

McKenna A, Hanna M, Banks E, Sivachenko A, Cibulskis K, Kernytsky A et al (2010) The genome analysis toolkit: a mapreduce framework for analyzing next-generation DNA sequencing data. Genome Res 20:1297-1303

Meng L, Li H, Zhang L, Wang J (2015) QTL IciMapping: integrated software for genetic linkage map construction and quantitative trait locus mapping in biparental populations. Crop J 3:269-283

Michelmore R, Paran I, Kesseli R (1991) Identification of markers linked disease-resistance genes by bulked segregant regions by using segregating populations. PNAS 88:9828-9832

Narusaka M, Hatakeyama K, Shirasu K, Narusaka Y (2014) Arabidopsisdual resistance proteins, both RPS4 and RRS1, are required for resistance to bacterial wilt in transgenic Brassicacrops. Plant Signal Behav 9:e29130

Norman DJ, Zapata M, Gabriel DW, Duan YP, Yuen JMF, MangravitaNovo A et al (2009) genetic diversity and host range variation of Ralstonia solanacearum strains entering North America. Phytopathology 99:1070-1077

Obrador-Sánchez JA, Tzec-Simá M, Higuera-Ciapara I, Canto-Canché B (2017) Genetic diversity of Ralstonia solanacearum strains from Mexico associated with Moko disease. Eur J Plant Pathol 149:817-830

Ouellette LA, Reid RW, Blanchard SG, Brouwer CR (2018) LinkageMapView rendering high-resolution linkage and QTL maps. Bioinform 34:306-307

Pandey MK, Wang ML, Qiao L, Feng S, Khera P, Wang H et al (2014) Identification of QTLs associated with oil content and mapping FAD2 genes and their relative contribution to oil quality in peanut (Arachis hypogaea L.). BMC Genet 15:133

Paudel S, Dobhal S, Alvarez AM, Arif M (2020) Taxonomy and phylogenetic research on Ralstonia solanacearum species complex: a complex pathogen with extraordinary economic consequences. Pathogens 9:886

Prasad M, Wang ML, Khera P, Pandey MK, Wang H, Qiao L et al (2015) Genetic mapping of QTLs controlling fatty acids provided insights into the genetic control of fatty acid synthesis pathway in peanut (Arachis hypogaea L.). PLoS ONE 10:e0119454

Qian YL, Wang XS, Wang DZ, Zhang LN, Zu CL, Gao ZL et al (2012) The detection of QTLs controlling bacterial wilt resistance in tobacco (N. tabacum L.). Euphytica 192:259-266

Robinson JT, Thorvaldsdóttir H, Winckler W, Guttman M, Lander ES, Getz $\mathrm{G}$ et al (2011) Integrative genomics viewer. Nat Biotechnol 29:24-26

Shasidhar Y, Vishwakarma MK, Pandey MK, Janila P, Variath MT, Manohar SS et al (2017) Molecular mapping of oil content and fatty acids using dense genetic maps in groundnut (Arachis hypogaea L.). Front Plant Sci 8:794

Suhandono S, Safitri R, Merry Hemelda N (2019) Genetic diversity of Ralstonia solanacearum, a phytopathogenic bacterium infecting horticultural plants in Java, Indonesia. Biodiversitas 20:364-372
Thorvaldsdottir H, Robinson JT, Mesirov JP (2012) Integrative Genomics Viewer (IGV): high-performance genomics data visualization and exploration. Brief Bioinf 14:178-192

Trick M, Adamski NM, Mugford SG, Jiang CC, Febrer M, Uauy C (2012) Combining SNP discovery from next-generation sequencing data with bulked segregant analysis (BSA) to fine-map genes in polyploid wheat. BMC Plant Biol 12:14

Tseng YC, Tillman BL, Peng Z, Wang J (2016) Identification of major QTLs underlying tomato spotted wilt virus resistance in peanut cultivar Florida-EPTM '113.' BMC Genet 17:128

Van Ooijen JW (2009) MapQTL ® 6, Software for the mapping of quantitative trait loci in experimental populations of diploid species. Kyazma B.V., Wageningen

van Ooijen JW (2018) JoinMap @ software for the calculation of genetic linkage maps in experimental populations of diploid species. Kyazma B.V., Wageningen

Vinatzer BA, Narusaka M, Kubo Y, Hatakeyama K, Imamura J, Ezura $\mathrm{H}$ et al (2013) Interfamily transfer of dual NB-LRR genes confers resistance to multiple pathogens. PLoS ONE 8:e55954

Voorrips RE (2002) MapChart: software for the graphical presentation of linkage maps and QTLs. J Hered 93:77-78

Wang L, Zhou X, Ren X, Huang L, Luo H, Chen Y et al (2018) A major and stable QTL for bacterial wilt resistance on chromosome B02 identified using a high-density SNP-based genetic linkage map in cultivated peanut Yuanza 9102 derived population. Front Genet 9:652

Xu J, Pan ZC, Prior P, Xu JS, Zhang Z, Zhang H et al (2009) Genetic diversity of Ralstonia solanacearum strains from China. Eur J Plant Pathol 125:641-653

Yu SL (2011) Chinese peanut genetics and breeding. Shanghai Science and Technology Press, Shanghai (in Chinese)

Zhang C, Chen H, Cai T, Deng Y, Zhuang R, Zhang N et al (2017) Overexpression of a novel peanut NBS-LRR gene AhRRS5 enhances disease resistance to Ralstonia solanacearumin tobacco. Plant Biotechnol J 15:39-55

Zhang S, Hu X, Miao H, Chu Y, Cui F, Yang W et al (2019) QTL identification for seed weight and size based on a high-density SLAF-seq genetic map in peanut (Arachis hypogaea L.). BMC Plant Biol 19:537

Zhao Y, Zhang C, Chen H, Yuan M, Nipper R, Prakash CS et al (2016) QTL mapping for bacterial wilt resistance in peanut (Arachis hypogaea L.). Mol Breed 36:13

Zhuang W, Chen H, Yang M, Wang J, Pandey MK, Zhang C et al (2019) The genome of cultivated peanut provides insight into legume karyotypes, polyploid evolution and crop domestication. Nat Genet 51:865-876

Publisher's Note Springer Nature remains neutral with regard to jurisdictional claims in published maps and institutional affiliations. 